\title{
Aplicación de la Cromatografía de Gases Bidimensional en el Análisis de Derivados Azufrados y Nitrogenados en Gasolina
}

María Ruiz-Guerrero ${ }^{1,2}$ y Fabrice Bertoncini ${ }^{1}$

(1) Institut Francais du Petrole, IFP-Lyon, BP, 69390 Vernaison-Francia

(2) IPN, Centro de Investigación en Biotecnología Aplicada Tlaxcala, San Juan Molino Km 1.5, Tepetitla, Tlaxcala-México (e-mail: maruizg@ipn.mx)

\section{Resumen}

Se emplea la Cromatografía de Gases Bidimensional (GC×GC) como una poderosa herramienta en el análisis de derivados con azufre y nitrógeno presentes en gasolinas. Se presentan los resultados obtenidos del acoplamiento de la GC×GC a un detector especifico, azufre (SCD) y nitrógeno (NCD). La técnica se basa en la separación en dos dimensiones ortogonales que son el resultado de la asociación de dos columnas de GC de diferente polaridad. El cromatograma final obtenido ésta estructurado en función de las propiedades de volatilidad y polaridad de los hidrocarburos, lo que facilita su identificación. Los resultados son muy prometedores en la caracterización de éstos compuestos en mezclas complejas. En este trabajo se ilustra el potencial que tiene el alto poder resolutivo de la GC×GC combinado con una detección selectiva de azufre y nitrógeno en la caracterización de gasolinas al utilizar un detector especifico para éstos contaminantes.

\section{Comprehensive Two-Dimensional Gas Chromatography Application in Sulphur and Nitrogen Speciation of Gasoline}

\begin{abstract}
The comprehensive two-dimensional gas chromatography ( $\mathrm{CC} \times \mathrm{GC})$ is used as a powerful tool for an improved characterization in sulphur and nitrogen speciation in gasoline. There are shown the results obtained of the connection between GC $\times G C$ and a specific detector, sulphur (SCD) and Nitrogen (NCD). This technique is based on the separation of two orthogonal dimensions, achieved by two chromatographic capillary columns with different polarity. The final chromatogram obtained was structured according to the volatility and polarity of hydrocarbons which facilitates its identification. The results are a very promising alternative in the characterization of these types of compounds in middle distillates. This work illustrates the high resolution power of the GC×GC in combination with a selective detection of sulphur or nitrogen compounds in gasoline when a specific detector is used.
\end{abstract}

Keywords: two-dimensional gas chromatography, specific detection, gasoline, nitrogen, sulphur 


\section{INTRODUCCION}

La reglamentación mundial del medio ambiente que regula los criterios sanitarios, ha resuelto orientar el mercado de los hidrocarburos hacia niveles aún más estrictos en el contenido de compuestos azufrados en gasolinas (compuestos-A). La norma internacional impuso que a partir de 2005 el azufre en gasolinas y diesel no debía exceder una concentración máxima de 10 ppm (0.05\%). Por otra parte, el contenido de compuestos nitrógenados (compuestos- $\mathrm{N}$ ) tienen un efecto adverso en la estabilidad del combustible e inhiben las reacciones catalíticas efectuadas durante el hidrotratamiento (Dorbon et al., 1989). Sin embargo, para mejorar este proceso, es necesario conocer la composición en moléculas azufradas y nitrogenadas presentes tanto en las cargas como en efluentes a fin de comprender los mecanismos de reacción que se llevan a cabo en el reactor. En el caso de los compuestos-A, ciertas especies como los dibenzotiofenos (DBT) y en menor medida los benzotiofenos (BT) son más refractarios al hidrotratamiento que los tiofenos ( $T$ ) o los mercaptanos, Figura 1. En particular, la substitución del dibenzotiofeno con grupos metilo en la posición 4 o 4,6 hace más lenta la reacción de desulfuración a causa del efecto estérico de ese grupo. La estructura química de diferentes familias de compuestos azufrados presentes en la gasolina se muestran en la figura 1.

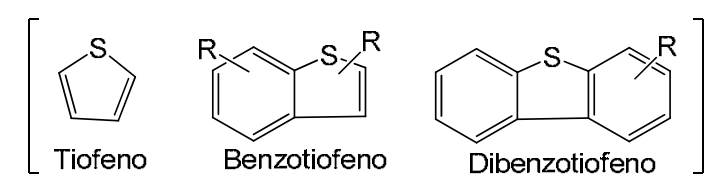

Fig. 1: Familias de compuestos azufrados presentes en gasolinas

En el caso de los compuestos- $N$, su remoción es altamente recomendada para asegurar una buena deshidrosulfuración (DHS). Además, debido a su baja concentración en gasolinas y complejidad de estructura, suele ser difícil su caracterización (Zeuthen et al., 2001). Los compuestos-N presentes en gasolinas se encuentran predominantemente en formas básicas (bencénicos de piridina) y forma neutras (bencénicos de pirrol) (Figura 2).

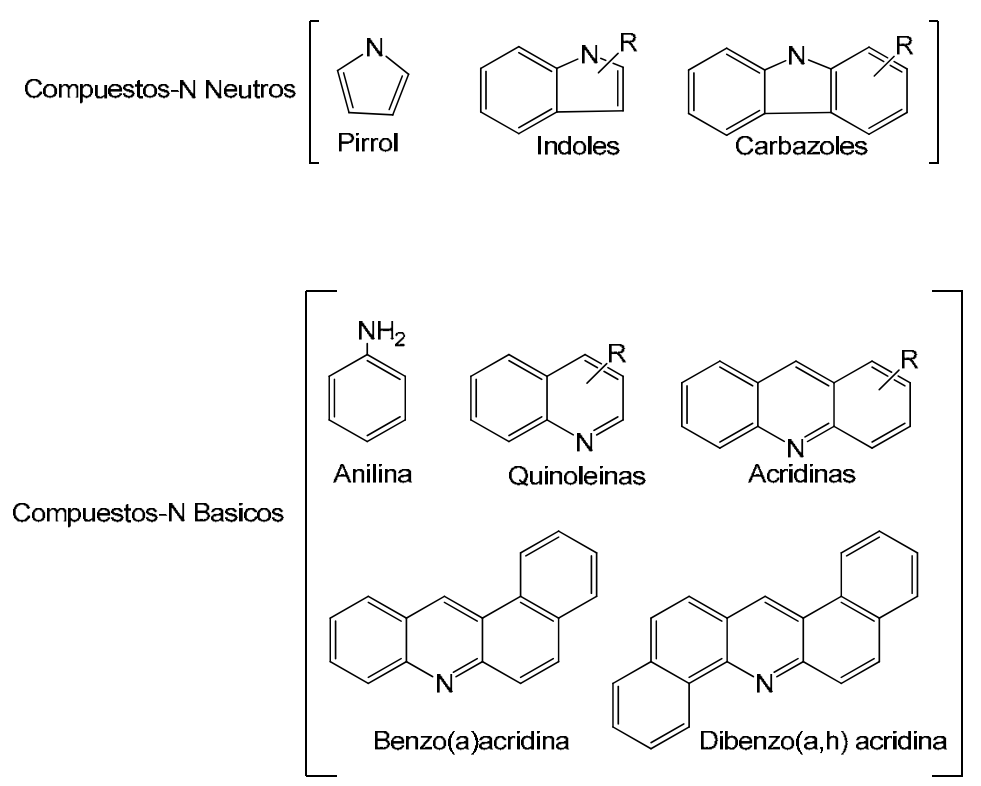

Fig. 2: Familias de compuestos nitrogenados presentes en gasolinas

Por lo anterior se ha incitado el desarrollo de nuevos métodos analíticos más sensibles y selectivos que permitan cuantificar este tipo de derivados en los productos petroleros. Los métodos más apropiados para determinar los contenidos de estos contaminantes son diversos, sin embargo; para acceder al detalle en la repartición de moléculas, ya sean compuestos con azufre ó nitrógeno, la cromatografía en fase gaseosa (GC) acoplada a un detector específico (azufre o nitrógeno, respectivamente) es la técnica generalmente más utilizada. El poder de resolución de la GC es sin 
embargo limitado, su principal inconveniente es el solapamiento de picos de elusión: para los compuestos-A los alquilbenzotiofenos (alquil-BT) pesados con los alquildibenzotiofenos (alquil-DBT) ligeros, y para los compuestos- $\mathrm{N}$, la moléculas neutras (indoles y carbazoles) con las básicas (piridinas y quinoleínas). Por otro lado, los cromatogramas en análisis independientes con detectores específicos para compuestos-A o compuestos- $\mathrm{N}$, de ciertas gasolinas, presentan un fondo continuo no resuelto que se ha atribuido de manera sistemática a determinados compuestos sin certeza.

En años recientes se ha diseñado una variación a la cromatografía de gases para la caracterización de mezclas complejas, la cromatografía de gases bidimensional (GC×GC). Esta técnica tiene hoy en día un lugar muy importante en la industria petrolera para el análisis de hidrocarburos ligeros, debido al alto poder de resolución que puede aportar (Ruiz-Guerrero et al., 2006; Vendeuvre et al., 2005a; Vendeuvre et al., 2005b; Wang et al., 2004). De manera general, las ventajas que resultan de la GC $\times$ GC son una resolución mejorada y alta sensibilidad, su principio y uso ha sido descrito (Vendeuvre et al., 2005b). Adicionalmente, el acoplamiento de la GC×GC a un detector especifico es una alternativa muy prometedora en la especiación de compuestos azufrados (Blomberg et al., 2003; Ruiz-Guerrero et al., 2006) ó nitrogenados (Adam et al., 2007; Wang et al., 2004), debido a que combina el alto poder resolutivo de la $G C \times G C$ y el aporte de una detección selectivas de azufre (GC×GC-SCD) o nitrógeno (GC×GC-NCD).

En este trabajo se muestra como el uso de un detector especifico acoplado a la GC $\times$ GC permite alcanzar una separación optima, siempre y cuando se cuente con una combinación apropiada de columnas de separación y un detector conforme al tipo de moléculas que se desee analizar. En este trabajo se muestra el aporte cuantitativo y cualitativo de la GC $\times$ GC adaptada a un detector selectivo de azufre y por otro lado el cualitativo a uno nitrógeno a fin de realizar el análisis de diferentes gasolinas. Se analiza su potencial comparativamente a la técnica convencional de GC. Los ejemplos de aplicación ilustran el alcance de la técnica en el análisis de gasolinas.

\section{MATERIALES Y MÉTODOS}

\section{Instrumentación compuestos Azufrados:}

Para los análisis de compuestos-A en CG-SCD, fué utilizado un detector modelo SCD Sievers 355B (SIEVERS Inc. Boulder, CO. USA), acoplado a un cromatógrafo HP 5890 Serie II (Agilent Tecnologies). Se uso una columna: DB1 $60 \mathrm{~m} \times 0.25 \mathrm{~mm}$ i.d.; $0.25 \mu \mathrm{m}$. (J\&W sintific., CA, USA), se inyectó $1.0 \mu \mathrm{l}$ se muestra a $270^{\circ} \mathrm{C}$. Programa de temperatura: $50-120^{\circ} \mathrm{C}, 10^{\circ} \mathrm{C} / \mathrm{min}, 1^{\circ} \mathrm{C} . / \mathrm{min}$ hasta $240^{\circ} \mathrm{C}, 10^{\circ} \mathrm{C} / \mathrm{min}$ hasta, $280^{\circ} \mathrm{C}(10 \mathrm{~min}), 200 \mathrm{kP}$ de presión para He como gas portador. El sistema bidimensional para el mismo detector (GC×GC-SCD) consiste de un cromatógrafo HP6890 GC (Agilent Technologies) acoplado a un detector SCD SIEVERS 355B. El modulador empleado y adaptado al cromatógrafo de gases, fue construido en laboratorio y consta de un modulador a dos válvulas con $\mathrm{CO}_{2}$ como gas enfriador, tal como es descrito por Beens et al. (2001). Una vez adquiridos los datos, estos fueron exportados a un archivo SCV y tratados posteriormente por un programa Matlab diseñado en casa (The Mathworks Natik, MA, USA) para su visualización y cuantificación. Las columnas empleadas en el sistema bidimensional: PONA, $10 \mathrm{~m} \times 0.2 \mathrm{~mm} \times 0.5 \mu \mathrm{m}$ (dimetilpolisiloxano, J\&W sientific, CA, USA ) y BPX50, $0.8 \mathrm{~m} \times 0.1 \mathrm{~mm}$ i.d (Fenil (equiv.) polisilfenilenesiloxano (50\% Ciano), SGE, Rongwood, Vic., Australia). Se inyectó $1.0 \mu \mathrm{l}$ en columna de muestra a $200 \mathrm{kP}$ de presión para el gas portador. Programa de temperatura: $60^{\circ} \mathrm{C}-280^{\circ} \mathrm{C} ; 2^{\circ} \mathrm{C} / \mathrm{min}$. Detector SCD: $1000^{\circ} \mathrm{C}$. La adquisición se llevo a cabo a $50 \mathrm{~Hz}$.

Muestras: Una mezcla sintética de compuestos azufrados con estándares puros, 46 compuestos en total, fue preparada para identificación de compuestos en una concentración entre 30 y 50 ppm w/w en tolueno ver Tabla 1. Además una mezcla de T, BT y DBT fué utilizada como estándar externo (Sigma Aldrich, Lyon, Francia).

La capacidad de la GC×GC en la identificación de los compuestos azufrados fue evaluada en dos diferentes tipos de gasolinas: Una gasolina proveniente de la conversión de productos más pesados a través del proceso conocido como craking catalítico (LCO) y una gasolina obtenida a través de la 
destilación directa -straight run- de petróleo bruto (SR), azufre total determinado por fluorescencia de rayos X. Método ASTM D2622, (p. eb. $150-347^{\circ} \mathrm{C}$ ). Las muestras fueron facilitadas por el centro de investigación del IFP (IFP, Vernaison, Francia) e inyectadas después de una dilución en tolueno de 100 a 400 ppm azufre total.

\section{Instrumentación compuestos Nitrogenados:}

El sistema bidimensional para el detector especifico a Nitrógeno (GC×GC-NCD) consiste de un cromatógrafo HP6890 GC (Agilent Technologies) acoplado a un detector NCD Antek 7090N (Antek instruments, Huston, TX, USA). El modulador empleado y adaptado al cromatógrafo de gases, es el mismo sistema que fue empleado en GC×GC-SCD y los datos fueron tratados de la misma manera. Las columnas empleadas en el sistema bidimensional: SPB-5, 30m $\times 0.25 \mathrm{~mm} \times 1.0 \mu \mathrm{m}(5 \%$ Fenil) metil polisiloxano, Supelco (St. Quentin-Fallavier, Francia) y BPX70, $1.0 \mathrm{~m} \times 0.1 \mathrm{~mm} \times 0.2 \mu \mathrm{m}(70 \%$ Ciano) propil polisilfenilene-siloxano, SGE (Courtaboeuf, France). Se inyectó $1.0 \mu$, en columna, de muestra a $200 \mathrm{kP}$ de presión para el gas portador. Programa de temperatura: $60^{\circ} \mathrm{C}-260^{\circ} \mathrm{C} ; 3^{\circ} \mathrm{C} / \mathrm{min}$. Detector NCD: $1000^{\circ} \mathrm{C}$. La adquisición se llevo a cabo a $50 \mathrm{~Hz}$.

Muestras: Una mezcla sintética de un gran numero de compuestos nitrogenados se preparó incluyendo 58 compuestos como estándares obtenidas de Chiron (Villeurbanne, Francia), Aldrich, (Lyon, Francia), Accros (Noisy le grand, Francia) en una concentración entre 18 y 24 ppm w/w (Tabla 2) y disueltos en una solución tolueno/acetona al $10 \%$. Una gasolina proveniente del craking catalítico (LCO2) fue proporcionada por el centro de investigación del IFP, 391 pmm contenido total $\mathrm{N}$; p. eb. $130-400^{\circ} \mathrm{C}$. Nitrógeno total por chemiluminiscencia de acuerdo al método ASTM D2629.

Tabla 1: Composición de la mezcla sintética de compuestos azufrados e identificación de picos

\begin{tabular}{|c|c|c|c|}
\hline No. & Compuesto & No. & Compuesto \\
\hline & Mercaptanos & 22 & 4-MeBT \\
\hline 1 & 1-Nonanetiol & 23 & 3-MeBT \\
\hline 2 & Isoamil sulfato & 25 & 2,4-DiMeBT \\
\hline 3 & 1-Decanetiol & 26 & 2,6-DiMeBT \\
\hline 4 & Hexil sulfuro & 27 & 3,5-DiMeBT \\
\hline 5 & 1-Dodecanotiol & 28 & 2,3-DiMeBT \\
\hline 6 & 1,9-Nonaneditiol & 29 & 2,5,7-TriMeBT \\
\hline 7 & 1-Pentadecanetiol & 30 & 2,3,4 TriMeBT \\
\hline 8 & Dioctisulfide & 31 & 2,3,6 TriMeBT \\
\hline 9 & 1-Hexadecanetiol & 32 & 2,3,4,7-TriMeBT \\
\hline & $T=T i o f e n o$ & & DBT=Dibenzotiofeno \\
\hline 10 & 2-n-PropilT & 33 & Dibenzotiofeno \\
\hline 11 & 2,3,5-TriMeT & 34 & 4-MeDBT \\
\hline 12 & 2-n-BuT & 35 & 2-MeDBT \\
\hline 13 & 3-n-BuT & 36 & 4-EtDBT \\
\hline 14 & 2-n-PentilT & 37 & 4,6-DiMeDBT \\
\hline 15 & 3-n-HexilT & 38 & 2,3-DiMeDBT \\
\hline 24 & 3-n-HeptilT & 39 & 2-EtDBT \\
\hline 16 & 3-PenilT- & 40 & 2,8-DiMeDBT \\
\hline 17 & 3-n-Octil & 41 & 2,4,6-TriMeDBT \\
\hline 18 & 2-n-OctilT & 42,43 & $2,4,8$ y $2,4,7$-TriMEDBT \\
\hline 19 & 3-n-OctadecilT & 44 & 2,3,8-TriMeDBT \\
\hline & BT=Benzotiofeno & 45 & 2-BuDBT \\
\hline 20 & Benzotiofeno & 46 & 2-PentilDBT \\
\hline 21 & 7-MeBT & & \\
\hline
\end{tabular}

\section{RESULTADOS Y DISCUSIÓN}

Compuestos-A: Una mezcla sintética de compuestos azufrados: sulfuros, tioles, tiofenos, benzotiofenos y dibenzotiofenos; susceptibles de estar presentes en las muestras de gasolinas y descritos en la Tabla 1, fue separada por GC×GC-SCD y es presentada en el cromatograma de la 
figura 3, la Tabla 1 también enumera estos compuestos de acuerdo al numero con que fue identificado en la figura 3. Este cromatograma muestra como con la GC ×GC-SCD es posible separar los compuestos, sobre el eje de las $X$ de acuerdo a su punto de ebullición, que se incrementa con el largo de la cadena; y sobre el eje de las $Y$ por polaridad, que se incrementa con el tipo y numero de anillos.

Tabla 2: Composición de la mezcla sintética de compuestos nitrogenados e identificación de picos

\begin{tabular}{|c|c|c|c|}
\hline No. & Compuesto & No. & Compuesto \\
\hline 1 & 2,6-Piridina & 30 & Indol \\
\hline 2 & 2 Et Piridina & 31 & 2-Me Indol \\
\hline 3 & 2 Et Pirrol & 32 & 7-Me Indol \\
\hline 4 & 2,4-DiMe Pirrol & 33 & 3-Me Indol \\
\hline 5 & 2,3-Piridina & 34 & 7- Et Indol \\
\hline 6 & 3 Et Piridina & 35 & 2,3-DiMe Indol \\
\hline 7 & 4 Et Piridina & 36 & 2,3,7-DiMe Indol \\
\hline 8 & 3,5-Piridina & 37 & 7,8-Benzo (h) Quinoleina \\
\hline 9 & Anilina & 38 & Acridina \\
\hline 10 & 1,2,5-TriMe Pirrol & 39 & 9-Et Carbazol \\
\hline 11 & 2,4,6-TriMe Pirrol & 40 & 9-Me Acridina \\
\hline 12 & 2,3,5-TriMe Pirrol & 41 & Carbazol \\
\hline 13 & 2-MeBenzonitrilo & 42 & 1-Me Carbazol \\
\hline 14 & 3-MeBenzonitrilo & 43 & 3-Me Carbazol \\
\hline 15 & 4-MeBenzonitrilo & 44 & 4-Me Carbazol \\
\hline 16 & 4 Et Anilina & 45 & 1,3-DiMe Carbazol \\
\hline 17 & 3 Et Anilina & 46 & 1,6-DiMe Carbazol \\
\hline 18 & Quinolina & 47 & 1,5-DiMe Carbazol \\
\hline 19 & Isoquinolina & 48 & 3,6-DiMe Carbazol \\
\hline 20 & 4-propil Anilina & 49 & 2,6-DiMe Carbazol \\
\hline 21 & 2-Me Quinolina & 50 & 2,7-DiMe Carbazol \\
\hline 22 & 8-Me Quinolina & 51 & 2,4-DiMe Carbazol \\
\hline 23 & 7-Me Quinoline & 52 & 2,5-DiMe Carbazol \\
\hline 24 & 6-Me Quinolina & 53 & 3,4-DiMe Carbazol \\
\hline 25 & 3-Me Quinolina & 54 & Benzo (a) Acridina \\
\hline 26 & 4-Me Quinolina & 55 & DiBenz (c,h) Acridina \\
\hline 27 & 1,2-DiMe Indol & 56 & DiBenz $(a, h)$ Acridina \\
\hline 28 & 2,6-DiMe Quinolina & 57 & DiBenz $(a, c)$ Acridina \\
\hline 29 & NNNN-TriMe Fenilendiamina & 58 & 7H-DiBenzo $(\mathrm{c}, \mathrm{g})$ Carbazol \\
\hline
\end{tabular}

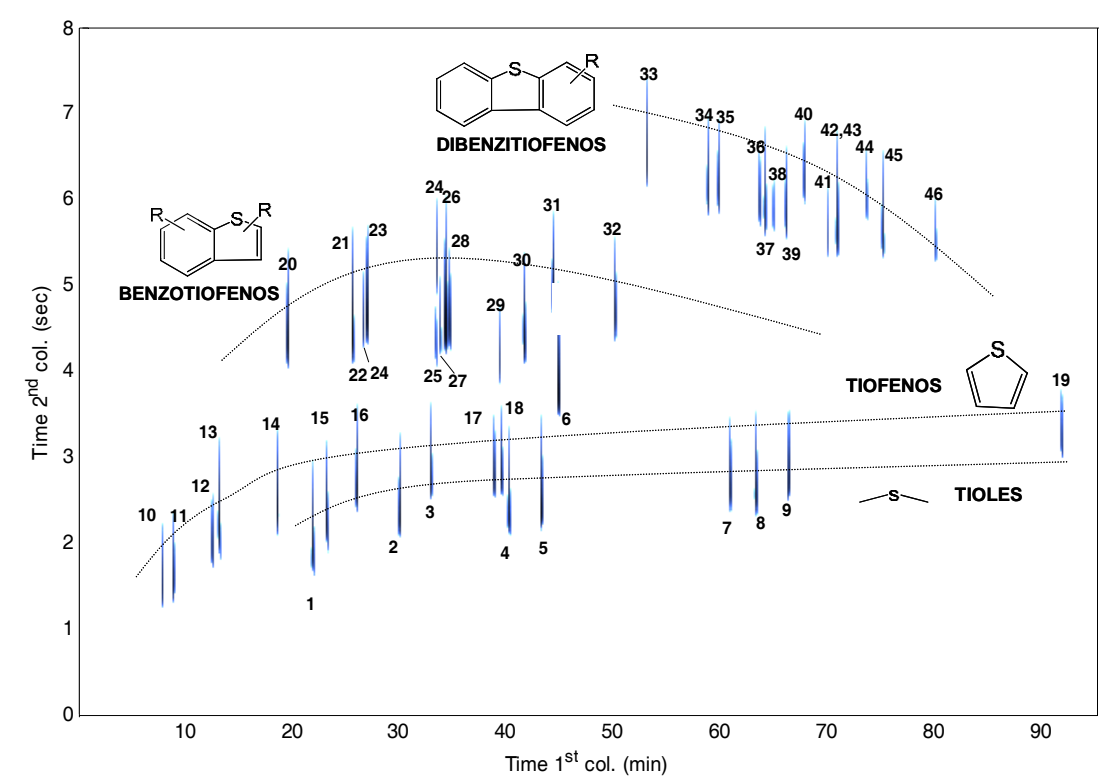

Fig. 3: Identificación de estándares azufrados por GC×GC-SCD. 
En la figura 3 se muestra además como la información que se obtiene aparece estructurada de acuerdo al grupo de familia-molécula que se trate: mercaptanos ó sulfuros, tiofenos, benzotiofenos y dibenzotiofenos. Una vez afinada la técnica de GC×GC-SCD, se empleo el mismo método de análisis para dos gasolinas comerciales LCO y SR. La figura 4, muestra un cromatograma clásico GC-SCD y el cromatograma bidimensional GC×GC-SCD para ambas muestras de gasolina. La gasolina LCO contiene básicamente compuestos tiofenicos, mientras que la muestra RS contiene compuestos tiofenicos y no tiofenicos con intenso solapamiento. Un análisis detallado, integrando los grupos o familia por números de carbono nos muestra para las gasolinas analizadas, que efectivamente para la LCO (Figura 5A) la relación BT/DBT es prácticamente igual tanto por la técnica GC-SCD como por GC $\times G C-S C D$, por otro lado para la gasolina SR (Figura 5B), que es una gasolina más compleja, la relación BT/DBT resulta ser muy diferente entre ambas técnicas, esta diferencia obedece a la imposibilidad de identificar y cuantificar estos compuestos en gasolinas complejas por GC-SCD.
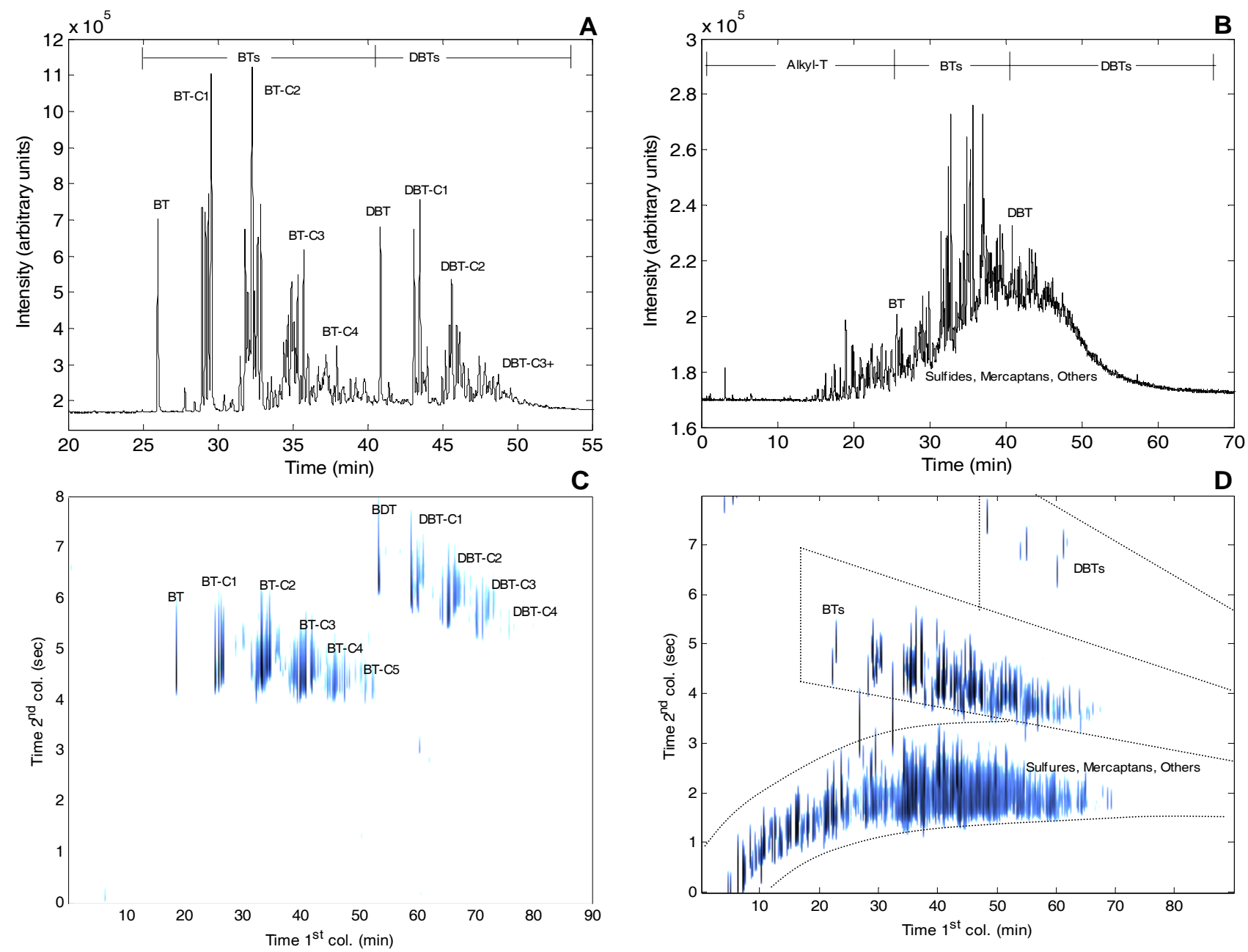

Fig.4: Perfiles cromatográficos por GC-SCD y GC×GC-SCD. Muestra LCO: A y C, muestra SR: B y $D$, respectivamente

Compuestos-N: Una mezcla sintética de compuestos nitrogenados; básicos y neutros; susceptibles de estar presentes en las muestras de gasolinas y descritos en la tabla 2, fue analizada por varios juegos de columnas con la finalidad de encontrar el juego de columnas propio para llevar a cabo una separación GC×GC-NCD. La figura 6, muestra el cromatograma de la mezcla sintética, obtenido por cromatografía bidimensional adaptada a un detector específico de nitrógeno. Los numerales de la figura corresponden a la identificación hecha en la tabla 2.

Al igual que en el caso de los compuestos- $\mathrm{A}$, los compuestos- $\mathrm{N}$ están perfectamente estructurados dentro del cromatograma, podemos observar en la figura 6 que en efecto, sobre el eje de las $X$ se tiene una separación de moléculas por punto de ebullición que aumenta al aumentar el número de átomos de carbono y sobre el eje de las $Y$ por polaridad. Encontramos las moléculas tipo pirrol o no 
básicos en la parte alta del cromatograma mientras que las moléculas tipo piridina o básicas en la parte baja. El mismo método se empleó para el análisis de una gasolina: LCO2. La figura 7, muestra el cromatograma bidimensional GC×GC-NCD para la muestra de gasolina, esta contiene en su mayor parte compuestos básicos: índoles y carbazoles como puede apreciarse en la figura. Una comparación entre los cromatogramas de la figura 6 de la mezcla sintética de componentes nitrogenados y la figura 7 correspondiente a la gasolina LCO2, demuestra una notable separación de compuestos en la gasolina, que permiten hacer una identificación por tipo de compuesto y número de átomos de carbonos o en algunas especies, hasta una identificación individual. Las líneas grises en las figuras 6 y 7 identifican los compuestos presentes
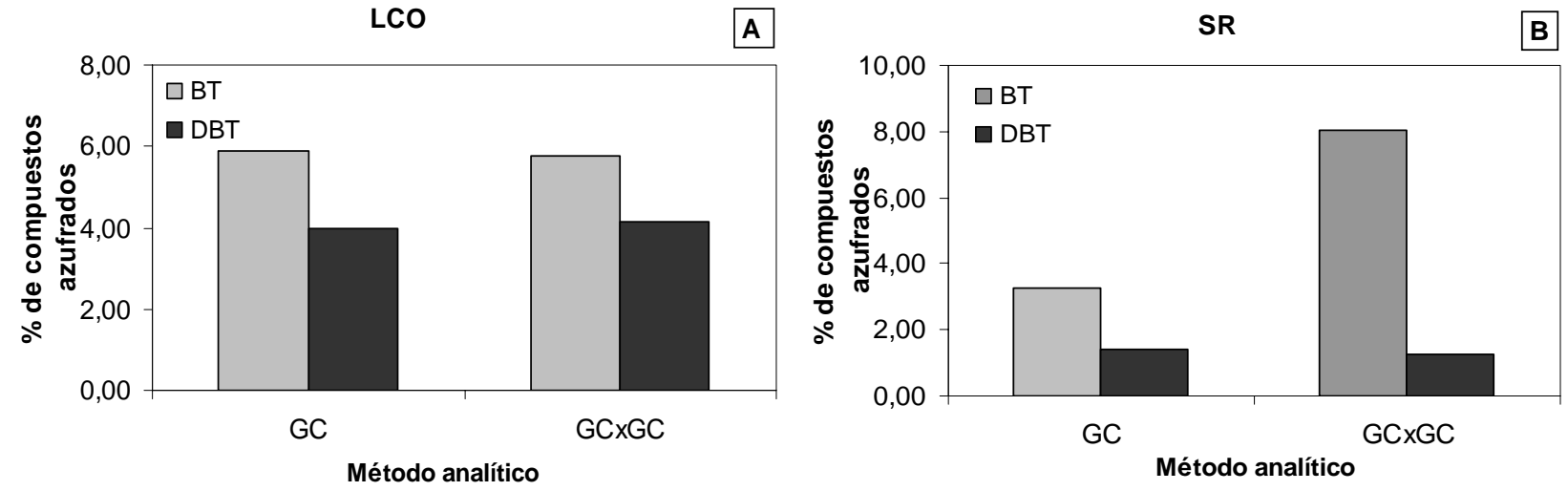

Fig. 5: Relación BT/DBT usando GC-SCD y GC×GC-SCD. A: gasolina LCO y B: gasolina SR

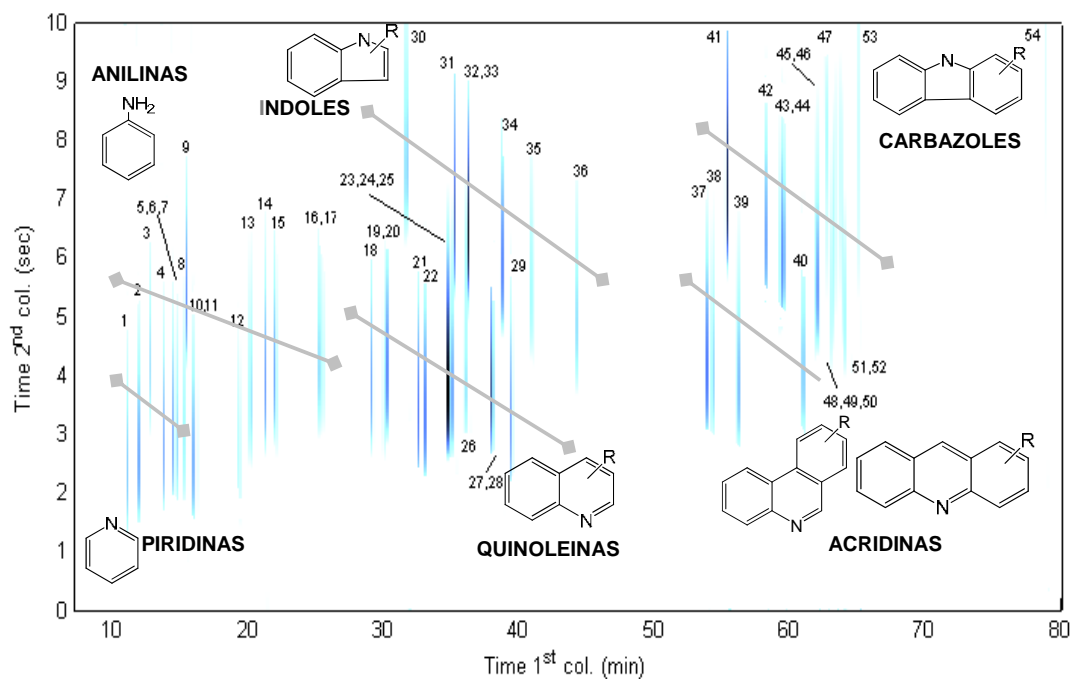

Fig. 6: Identificación de estándares nitrogenados (EST2) por GC×GC-NCD

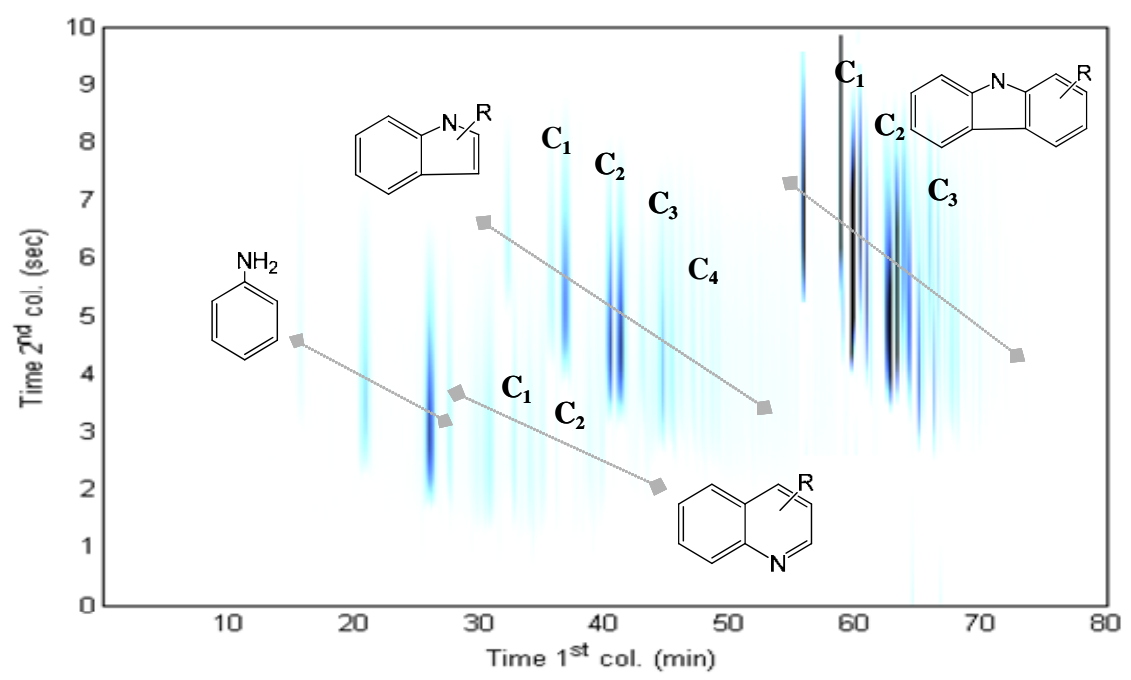

Fig. 7: Perfil cromatográfico por GC $\times$ GC-NCD para la muestra de gasolina LCO2 


\section{CONCLUSIONES}

La comparación que se hace entre la técnica convencional de GC y la GC×GC cuando se analizan compuestos azufrados permite comprobar que esta última, es una técnica valiosa en el análisis de mezclas complejas como son las gasolinas. Los resultados obtenidos por medio de la cromatografía de gases bidimensional nos permiten ir más lejos en la especiación de moléculas que por la técnica ordinaria, no es posible identificar. Además, resuelve solapamientos inciertos que la GC convencional no hace. La GC×GC permite también una excelente identificación de moléculas nitrogenadas, lo que nos permite una vez más afirmar la superioridad de esta en cuanto a mejor resolución, sensibilidad y especiación tanto en compuesto azufrados como nitrogenados.

\section{REFERENCIAS}

Adam, F. y otros seis autores; New benchmark for basic and neutral nitrogen compounds speciation in middle distillates using comprehensive two-dimensional gas chromatograph, J. Chromatogr.: 1148, 55-64 (2007).

Beens, J. y otros cuatro autores; Simple, non-moving modulation interface for comprehensive twodimensional gas chromatography, J. Chromatogr. A: 119, 127-132 (2001).

Blomberg, T. y otros tres autores;Comprehensive two-dimensional gas chromatography coupled with fast sulphur-chemiluminescence detection: implications of detector electronics, J. Chromatogr. A: 1050, 77-84 (2003).

Dorbon, M. y C. Bernasconi; Nitrogen compounds in light cycle oils: identification and consequences of ageing, Fuel: 68, 1067-1074 (1989).

Ruiz-Guerrero, R. y otros cuatro autores; Comparison of comprehensive two-dimensional gas chromatography coupled with sulfur-chemiluminescence detector to standard methods for speciation of sulfur-containing compounds in middle distillates, J. Chromatogr. Sci.: 44, 566-573 (2006).

Vendeuvre, C. y otros cuatro autores; Multidimensional gas chromatography for the detailed PIONA analysis of heavy naphtha: Hyphenation of an olefin trap to comprehensive two-dimensional gas chromatography, Journal of Chromatography A: 1090, 116-125 (2005a).

Vendeuvre, C. y otros cinco autores; Characterisation of middle-distillates by comprehensive twodimensional gas chromatography (GC $\times$ GC): A powerful alternative for performing various standard analysis of middle-distillates, Journal of Chromatography A: 1086, 21-28 (2005b).

Wang, F. C., W. K. Robbins y M. A. Greaney; Speciation of nitrogen-containing compounds in diesel fuel by comprehensive two-dimensional gas chromatography, J. Sep, Sci.: 27, 468-472 (2004).

Zeuthen, P., K. G. Knudsen y D. D. Whitehurst; Organic nitrogen compounds in gas oil blends, their hydrotreated products and the importance to hydrotreatment, Catalysis Today: 65, 307-314 (2001). 